PROCEEDINGS OF THE

AMERICAN MATHEMATICAL SOCIETY

Volume 140, Number 7, July 2012, Pages 2551-2560

S 0002-9939(2011)11094-3

Article electronically published on November 18, 2011

\title{
BORSUK-ULAM TYPE THEOREMS FOR MANIFOLDS
}

\author{
OLEG R. MUSIN
}

(Communicated by Alexander N. Dranishnikov)

\begin{abstract}
This paper establishes a Borsuk-Ulam type theorem for PLmanifolds with a finite group action, depending on the free equivariant cobordism class of a manifold. In particular, necessary and sufficient conditions are considered for a manifold with a free involution to be of Borsuk-Ulam type.
\end{abstract}

\section{INTRODUCTION}

The Borsuk-Ulam theorem states that any continuous antipodal $f: \mathbb{S}^{n} \rightarrow \mathbb{R}^{n}$ has zeros. (A map $f$ is called antipodal if $f(-x)=-f(x)$.) One of the most interesting proofs of this theorem is Bárány's geometric proof [1] (see also [9, Sec. 2.2]). A very similar proof was given in [10, and [13] has several more references for proofs of this type.

Let $X=\mathbb{S}^{n} \times[0,1], X_{0}=\mathbb{S}^{n} \times\{0\}$, and $X_{1}=\mathbb{S}^{n} \times\{1\}$. Let $\tau(x, t)=(-x, t)$, where $(x, t) \in X, x \in \mathbb{S}^{n}$, and $t \in[0,1]$. Clearly, $\tau$ is a free involution on $X$.

The first step of Bárány's proof is to show that any continuous antipodal (i.e. $F(\tau(x))=-F(x))$ map $F: X \rightarrow \mathbb{R}^{n}$ can be approximated by "sufficiently generic" antipodal maps (see [9, Sec. 2.2]).

Let $f_{i}: \mathbb{S}^{n} \rightarrow \mathbb{R}^{n}$, where $i=0,1$, be antipodal generic maps. Let $F(x, t)=$ $t f_{1}(x)+(1-t) f_{0}(x)$. Since $F$ is generic, the set $Z_{F}:=F^{-1}(0)$ is a manifold of dimension one. Then $Z_{F}$ consists of $\operatorname{arcs}\left\{\gamma_{k}\right\}$ with ends in $Z_{f_{i}}:=Z_{F} \cap X_{i}=f_{i}^{-1}(0)$ and cycles which do not intersect $X_{i}$. Note that $\tau\left(Z_{F}\right)=Z_{F}$ and $\tau\left(\gamma_{i}\right)=\gamma_{j}$ with $i \neq j$. Therefore, $\left(Z_{F}, Z_{f_{0}}, Z_{f_{1}}\right)$ is a $\mathbb{Z}_{2}$-cobordism. It is not hard to see that $Z_{f_{0}}$ is $\mathbb{Z}_{2}$-cobordant to $Z_{f_{1}}$ if and only if $\left|Z_{f_{1}}\right|=\left|Z_{f_{0}}\right|(\bmod 4)$.

To complete the proof, take $f_{0}$ as the standard orthogonal projection of $\mathbb{S}^{n}$ onto $\mathbb{R}^{n}$ :

$$
f_{0}\left(x_{1}, \ldots, x_{n}, x_{n+1}\right)=\left(x_{1}, \ldots, x_{n}\right), \text { where } x_{1}^{2}+\ldots+x_{n+1}^{2}=1 .
$$

Since $\left|Z_{f_{0}}\right|=2$, we have $\left|Z_{f_{1}}\right|=2(\bmod 4)$. This equality shows that for any antipodal generic $f_{1}$ the set $Z_{f_{1}}=f_{1}^{-1}(0)$ is not empty.

Our analysis of this proof shows that it can be extended for a wide class of manifolds. For instance, consider two-dimensional orientable manifolds $N=M_{g}^{2}$ of even genus $g$ and nonorientable manifolds $N=P_{m}^{2}$ with even $m$. Without loss of generality, we can assume that $N$ is "centrally symmetric" embedded to $\mathbb{R}^{k}$, where $k=3$ for $N=M_{g}^{2}$ and $k=4$ for $N=P_{m}^{2}$. That means $A(N)=N$, where

Received by the editors December 4, 2009 and, in revised form, May 24, 2010; December 18, 2010; January 13, 2011; January 18, 2011; January 23, 2011; and February 24, 2011.

2010 Mathematics Subject Classification. Primary 55M35, 55M99, 57R85.

Key words and phrases. Borsuk-Ulam theorem, group action, equivariant cobordism.

This research was supported in part by NSF grant DMS-0807640 and NSA grant MSPF-08G201.

(C)2011 American Mathematical Society 2551

Reverts to public domain 28 years from publication 
$A(x)=-x$ for $x \in \mathbb{R}^{k}$. Then $T:=\left.A\right|_{N}: N \rightarrow N$ is a free involution. It can be shown that there is a projection of $N \subset \mathbb{R}^{k}$ into a 2-plane $R$ passing through the origin 0 with $\left|Z_{f_{0}}\right|=2$. (See the details in Corollary 1.)

Actually, Bárány's proof can be (almost word for word) applied for $N$. Namely, the following statement holds:

Let $N=M_{g}^{2}$ with even $g$ or $N=P_{m}^{2}$ with even $m$. Then for any continuous $h: N \rightarrow \mathbb{R}^{2}$ there is $x \in N$ such that $h(T(x))=h(x)$.

Note that $N$ can be represented as a connected sum $M \# M$. This statement can be extended for $N=M \# M$ with any closed manifold $M$ (see Corollary 1) 11 We see that one of the most important steps here is the existence of a generic equivariant $f_{0}: N^{n} \rightarrow \mathbb{R}^{n}$ with $\left|Z_{f_{0}}\right|=2(\bmod 4)$.

This approach works for any free group action. Namely, for both technical steps, a "generic approximation lemma" and a " $\left(Z_{F}, Z_{f_{0}}, Z_{f_{1}}\right)$ cobordism lemma" can be extended for free actions of a finite group $G$ (Section 2).

Let $G$ be a finite group acting free on a closed connected PL-manifold $M^{m}$ and linearly on $\mathbb{R}^{n}$. In Section 3 we show a Borsuk-Ulam type theorem for $M$, depending on the free equivariant cobordism class of $M$ (Theorem 1). For the case $m=n$, Theorem 1 shows that if there is a continuous equivariant transversal to zeros $h: M^{n} \rightarrow \mathbb{R}^{n}$ with $\left|Z_{h}\right|=|G|(\bmod 2|G|)$, then for any continuous equivariant $f: M^{n} \rightarrow \mathbb{R}^{n}$, the zero set $Z_{f}$ is not empty.

In Section 4, this approach is applied to the classical case: manifolds with free involutions. There we give necessary and sufficient conditions for $\mathbb{Z}_{2}$-manifolds to have a Borsuk-Ulam type theorem (Theorem 2).

Lemma 4 shows that the zero set $Z_{f}$ is invariant up to $G$-cobordisms. Based on this fact, in Section 5 we define homomorphisms $\mu$ of free $G$-cobordism groups which can be considered as obstructions for $G$-maps. Theorem 3 shows that for a free $G$ manifold $M^{m}$ and a given linear action $G$ on $\mathbb{R}^{n}$, if this invariant is not zero in the free equivariant cobordisms, then for any continuous equivariant $f: M^{m} \rightarrow \mathbb{R}^{n}$ the set $Z_{f}$ is not empty. In this section we also provide some applications of Theorem 3 for the case $G=\left(\mathbb{Z}_{2}\right)^{k}$.

The main goal of this paper is to show that the geometric proof gives a method for checking whether a $G$-manifold $M$ is of the Borsuk-Ulam type. Namely, from Theorem 1 and its extension, Corollary 2, it follows that if there exists a generic equivariant $h: M^{m} \rightarrow \mathbb{R}^{n}$ with $\left[Z_{h}\right] \neq 0$ in the correspondent group of cobordisms, then for any continuous equivariant $f: M^{m} \rightarrow \mathbb{R}^{n}$ the set of zeros $Z_{f} \neq \emptyset$.

\section{Generic $G$-MAPS}

If $G$ is a group and $X$ is a set, then a group action of $G$ on $X$ is a binary function $G \times X \rightarrow X$ denoted by $(g, x) \rightarrow g(x)$ which satisfies the following two axioms: (1) $(g h)(x)=g(h(x))$ for all $g, h$ in $G$ and $x$ in $X ;(2) e(x)=x$ for every $x$ in $X$ (where $e$ denotes the identity element of $G$ ). The set $X$ is called a $G$-set. The group $G$ is said to act on $X$.

Let a finite group $G$ act on a set $X$. We say that $Y \subset X$ is a $G$-subset if $g(y) \in Y$ for all $g \in G$ and $y \in Y$, i.e. $G(Y)=Y$. Clearly, for any $x \in X$ the orbit $G(x):=\{g(x), g \in G\}$ is a $G$-subset. Denote by $\operatorname{Orb}(X, G)$ the set of orbits of $G$ on $X$, i.e. $\operatorname{Orb}(X, G)=\{G(x)\}$.

\footnotetext{
${ }^{1}$ It is not hard to prove the corollary using the standard techniques developed in [4]. However, we could not find this kind of statement in the Borsuk-Ulam theorem literature.
} 
Let $G_{x}:=\{g \in G \mid g(x)=x\}$ denote the stabilizer (or isotropy subgroup). Let $X^{H}=\{x \in X \mid g(x)=x, \forall g \in H\}$ denote the fixed point set of a subgroup $H \subset G$. If $H$ is isomorphic to $G_{x} \neq e$, then $X^{H} \neq \emptyset$.

Recall that a group action is called free if $G_{x}=\{e\}$ for all $x$; i.e. $g(x)=x$ if and only if $g=e$. Note that for a free action $G$ on $X$ each orbit $G(x)$ consists of $|G|$ points.

Here we consider piecewise linear (or $P L$, or simplicial) $G$-manifolds $X$; i.e. there is a triangulation $\Lambda$ of $X$ such that for any $g \in G$, any simplex $\sigma$ of $\Lambda$ is mapped bijectively onto the simplex $g(\sigma)$. The triangulation $\Lambda$ is called equivariant. Actually, any smooth $G$-manifold admits an equivariant triangulation [8].

Every free action of a finite group $G$ on a compact PL-manifold $X$ admits an equivariant triangulation such that for each simplex there are no vertices in the same orbit. Otherwise, we indeed can subdivide these simplices.

A map $f: X \rightarrow Y$ of $G$-sets $X$ and $Y$ is called equivariant (or a $G$-map) if $f(g(x))=g(f(x))$ for all $g \in G, x \in X$.

For a PL $G$-space (in particular, for a PL $G$-manifold) we say that an equivariant map $f: X \rightarrow \mathbb{R}^{n}$ is simplicial if $f$ is a linear map for each simplex $\sigma \in \Lambda$. For an equivariant triangulation $\Lambda$, any simplicial map $f: X \rightarrow \mathbb{R}^{n}$ is uniquely determined by the set of vertices $V(\Lambda)$. Indeed, for each simplex $\sigma$ of $\Lambda, f$ is linear and therefore is determined by the vertices of $\sigma$.

Any equivariant continuous map can be approximated by an equivariant simplicial map.

Lemma 1. Let $G$ be a finite group acting linearly on $\mathbb{R}^{n}$. Let $X$ be a compact simplicial G-space. Then for any equivariant continuous $f: X \rightarrow \mathbb{R}^{n}$ and $\varepsilon>0$, there is an equivariant simplicial map $\bar{f}: X \rightarrow \mathbb{R}^{n}$ such that $\|f(x)-\bar{f}(x)\| \leq \varepsilon$ for all $x \in X$.

Proof. It is well known (see, for instance, [7, Section 2.2]) that any continuous map on a compact PL-complex can be approximated by simplicial maps. Therefore there exists a triangulation $\Lambda$ and a simplicial map $\hat{f}$ such that $\|f(x)-\hat{f}(x)\| \leq \varepsilon$ for all $x \in X$. Clearly, there exists an equivariant subdivision $\Lambda^{\prime}$ of $\Lambda$ with $G(V(\Lambda)) \subset$ $V\left(\Lambda^{\prime}\right)$. For $v \in V\left(\Lambda^{\prime}\right)$, let $\bar{f}(v):=f(v)$. Then $\left.\bar{f}\right|_{V\left(\Lambda^{\prime}\right)}$ defines a simplicial map with $\|f(x)-\bar{f}(x)\| \leq \varepsilon$.

Let $\rho: G \rightarrow \operatorname{GL}(n, \mathbb{R})$ be a representation of a group $G$ on $\mathbb{R}^{n}$. In other words, $G$ is acting linearly on $\mathbb{R}^{n}$. Then $F:=\left(\mathbb{R}^{n}\right)^{G}$ is a linear subspace of $\mathbb{R}^{n}$. Denote by $L$ an invariant linear subspace of $\mathbb{R}^{n}$ which is transverse to $F$. Then $L^{G}=\{0\}$. So without loss of generality we can consider only linear actions with $\left(\mathbb{R}^{n}\right)^{G}=\{0\}$, i.e. linear actions on $\mathbb{R}^{n}$ such that the fixed point set of these actions is the origin.

Let $f: X \rightarrow \mathbb{R}^{n}$. Denote by $Z_{f}$ the set of zeros, i.e.

$$
Z_{f}=\{x \in X: f(x)=0\} .
$$

In this paper we need generic maps with respect to $Z_{f}$. Let $X$ be a simplicial $m$ dimensional $G$-manifold. Set $O_{\varepsilon}:=\left\{v \in \mathbb{R}^{n}:\|v\|<\varepsilon\right\}$. A continuous equivariant $f: X^{m} \rightarrow \mathbb{R}^{n}$ is called transversal to zeros if $Z_{f}$ is a manifold of dimension $m-n$, and there is an $\varepsilon>0$ such that for any $v \in O_{\varepsilon}$, the sets $f^{-1}(v)$ and $Z_{f}$ are homeomorphic.

Definition. Given a finite group $G$, let $X^{m}$ be a PL free $G$-manifold. Let $\Lambda$ be an equivariant triangulation of $X$. We say that an equivariant simplicial map 
$f: X \rightarrow \mathbb{R}^{n}$ is generic (with respect to zeros) if $Z_{f}$ does not intersect the $(n-1)$ skeleton $\Lambda(n-1)$ of $\Lambda$. (Recall that the $k$-skeleton of $\Lambda$ is the subcomplex $\Lambda(k)$ that consists of all simplices of dimension at most $k$.)

All simplicial generic maps are transversal to zeros.

Lemma 2. Let $G$ be a finite group acting linearly on $\mathbb{R}^{n}$ with $\left(\mathbb{R}^{n}\right)^{G}=\{0\}$. Let $X^{m}$ be a PL compact $m$-dimensional free $G$-manifold with or without boundary. Let $f: X \rightarrow \mathbb{R}^{n}$ be an equivariant simplicial generic map. If $n \leq m$, then $Z_{f}$ is an invariant submanifold of $X$ of dimension $m-n$. Moreover, if the boundary $\partial Z_{f}$ is not empty, then it lies in $\partial X$.

Proof. Note that $Z_{f} \subset X$ is a locally polyhedral surface consisting of $(m-n)$ dimensional cells. This is because for each $m$-simplex $\sigma$ we have the linear map $\left.f\right|_{\sigma}: \sigma \rightarrow \mathbb{R}^{n}$, and $Z_{f} \cap \sigma$ is defined by the generic linear equation $f(x)=0$. Hence, the components of $Z_{f}$ are PL-manifolds.

For $G$-spaces, the Tietze-Gleason theorem states: Let a compact group $G$ act on $X$ with a closed invariant set $A$. Let $G$ also act linearly on $\mathbb{R}^{n}$. Then any equivariant $f: A \rightarrow \mathbb{R}^{n}$ extends to $f: X \rightarrow \mathbb{R}^{n}$ (see [3, Theorem 2.3]).

It is known from results of Bierstone [2] and Field [6] on $G$-transversality theory that the zero set is a stratified set. If $G$ acts freely on a compact smooth $G$ manifold $X$, then this theory implies that the set of nongeneric equivariant smooth $f: X^{m} \rightarrow \mathbb{R}^{n}$ has measure zero in the space of all smooth $G$-maps. Let us extend this result as well as the Tietze-Gleason theorem for the PL case.

Lemma 3. Let $G$ be a finite group acting linearly on $\mathbb{R}^{n}$ with $\left(\mathbb{R}^{n}\right)^{G}=\{0\}$. Let $X^{m}$ be a PL compact free $G$-manifold with or without boundary. Let $\Lambda$ be an equivariant triangulation of $X$. Let $A$ be an invariant subset of $V(\Lambda)$ or let $A=\emptyset$. Let $h: A \rightarrow \mathbb{R}^{n}$ be a given equivariant generic map. Then the set of nongeneric simplicial maps $f: X \rightarrow \mathbb{R}^{n}$ with $\left.f\right|_{A}=h$ has measure zero in the space of all possible equivariant maps $f: V(\Lambda) \rightarrow \mathbb{R}^{n}$ with $\left.f\right|_{A}=h$.

Proof. Let $V^{\prime}:=V(\Lambda) \backslash A$. Let $C \in \operatorname{Orb}\left(V^{\prime}, G\right)$ be an orbit of $G$ on $V^{\prime}$. Then $|C|=p$, where $p:=|G|$. Since for any equivariant $f: V(\Lambda) \rightarrow \mathbb{R}^{n}$ and $v \in V(\Lambda)$ we have $f(g(v))=g(f(v))$, the vector $u=f(v) \in \mathbb{R}^{n}$ yields the map $f: C \rightarrow \mathbb{R}^{n}$.

Suppose $G$ has $k$ orbits on $V^{\prime}$. Take in each orbit $C_{i} \in \operatorname{Orb}\left(V^{\prime}, G\right)$ a vertex $x_{i}$. Denote by $M_{G}$ the space of all equivariant maps $f: V^{\prime} \rightarrow \mathbb{R}^{n}$. Then the space $M_{G}$ is of dimension $N=k n$.

Consider an $(n-1)$-simplex $\sigma \in \Lambda$ with vertices $v_{1}, \ldots, v_{n}$ which are not all from $A$. Note that $f \in M_{G}$ is not generic on $\sigma$ if $0 \in f(\sigma)$. In particular, the hyperplane in $\mathbb{R}^{n}$ which is defined by vectors $f\left(v_{1}\right), \ldots, f\left(v_{n}\right)$ passes through the origin 0 . In other words, the determinant of $n$ vectors $f\left(v_{1}\right), \ldots, f\left(v_{n}\right)$ equals zero.

This constraint gives a proper algebraic subvariety $s(\sigma)$ in $M_{G}$. Let $v_{1}, \ldots, v_{d}$ be vertices of $\sigma$ which are not in $A$. Then $s(\sigma)$ is of dimension $N-1$. Indeed, $u_{i}=f\left(v_{i}\right), i=1, \ldots, d$, are vectors in $\mathbb{R}^{n}$. Then the equation

$$
\operatorname{det}\left(u_{1}, \ldots, u_{d}, h\left(v_{d+1}\right), \ldots, h\left(v_{n}\right)\right)=0
$$

defines the subvariety $s(\sigma)$ in $M_{G}$ of dimension $N-1$.

Since the union of all subvarieties $s(\sigma)$ with $\operatorname{dim} \sigma=n-1$ is at most of dimension $N-1$, it has measure zero in $M_{G}$. From this it follows that the set of all nongeneric $f$ has measure zero in this space. 
Let $M_{0}$ and $M_{1}$ be closed $m$-dimensional simplicial manifolds with free actions of a finite group $G$. Then an $(m+1)$-dimensional simplicial free $G$-manifold $W$ is called a free $G$-cobordism $\left(W, M_{0}, M_{1}\right)$ if the boundary $\partial W$ consists of $M_{0}$ and $M_{1}$, and the action $G$ on $W$ respects actions on $M_{i}$.

Lemma 4. Let $G$ be a finite group acting linearly on $\mathbb{R}^{n}$ with $\left(\mathbb{R}^{n}\right)^{G}=\{0\}$. Let $\left(W, M_{0}, M_{1}\right)$ be a free $G$-cobordism. Let $f_{i}: M_{i}^{m} \rightarrow \mathbb{R}^{n}, i=0,1$, be equivariant simplicial generic maps. Then there is an equivariant simplicial generic $F: W \rightarrow$ $\mathbb{R}^{n}$ with $\left.F\right|_{M_{i}}=f_{i}$ and such that $\left(Z_{F}, Z_{f_{0}}, Z_{f_{1}}\right)$ is a free $G$-cobordism.

Proof. The existence of such a generic $F$ follows from Lemma 3 Lemma 2 implies that $Z_{F}$ is a $G$-submanifold of $W$. Clearly, $g\left(Z_{F}\right)=Z_{F}$ and $g\left(Z_{f_{i}}\right)=Z_{f_{i}}$ for all $g \in G$. Since $\partial Z_{F}=Z_{f_{0}} \sqcup Z_{f_{0}}$ is an $(m-n)$-dimensional $G$-manifold, we have a free $G$-cobordism $\left(Z_{F}, Z_{f_{0}}, Z_{f_{1}}\right)$.

\section{3. $G$-MAps AND A Borsuk-Ulam TYPE THEOREM}

In this section we consider a Borsuk-Ulam type theorem for the case $m=n$.

Definition. Given a finite group $G$ acting freely on a closed PL-manifold $M^{n}$ and acting linearly on $\mathbb{R}^{n}$ with $\left(\mathbb{R}^{n}\right)^{G}=\{0\}$, let $f: M^{n} \rightarrow \mathbb{R}^{n}$ be a continuous equivariant transversal to zeros map. Since $Z_{f}$ is a finite free $G$-invariant subset of $M$, we have $\left|Z_{f}\right|=k|G|$, where $k=0,1,2, \ldots$ Set $\operatorname{deg}_{G}(f):=1$ if $k$ is odd, and $\operatorname{deg}_{G}(f):=0$ if $k$ is even.

Lemma 5. Let $G$ be a finite group acting linearly on $\mathbb{R}^{n}$ with $\left(\mathbb{R}^{n}\right)^{G}=\{0\}$. Let $\left(W^{n+1}, M_{0}^{n}, M_{1}^{n}\right)$ be a free $G$-cobordism. Let $h_{i}: M_{i}^{n} \rightarrow \mathbb{R}^{n}, i=0,1$, be continuous equivariant transversal to zeros maps. Then $\operatorname{deg}_{G}\left(h_{0}\right)=\operatorname{deg}_{G}\left(h_{1}\right)$.

Proof. Lemma 1 and Lemma 3 yield that for any $h_{i}: M_{i}^{n} \rightarrow \mathbb{R}^{n}$ and $\varepsilon>0$ there is a generic map $a_{i, \varepsilon}$ such that $\left\|a_{i, \varepsilon}(x)-h_{i}(x)\right\| \leq \varepsilon$ for all $x \in M_{i}$. If $\varepsilon \rightarrow 0$, then $Z_{i, \varepsilon}:=a_{i, \varepsilon}^{-1}(0) \rightarrow Z_{h_{i}}$. This implies that for a sufficiently small $\varepsilon$ there is an equivariant bijection between $Z_{i, \varepsilon}$ and $Z_{h_{i}}$. Therefore, $\operatorname{deg}_{G}\left(a_{i, \varepsilon}\right)=\operatorname{deg}_{G}\left(h_{i}\right)$.

Let $\varepsilon$ be sufficiently small. Set $f_{i}:=a_{i, \varepsilon}$. From Lemma 4 it follows that there is an equivariant simplicial generic $F: W \rightarrow \mathbb{R}^{n}$ with $\left.F\right|_{M_{i}}=f_{i}$ such that $\left(Z_{F}, Z_{f_{0}}, Z_{f_{1}}\right)$ is a free $G$-cobordism. We have $m=n$. Then $Z_{F}$ is a submanifold of $W$ of dimension one. Therefore $Z_{F}$ consists of $\operatorname{arcs} \gamma_{1}, \ldots, \gamma_{\ell}$ with ends in $Z_{f_{i}}$ and cycles which do not intersect $M_{i}$.

Since any continuous map $s: \gamma_{k} \rightarrow \gamma_{k}$ has a fixed point, $G$ cannot act freely on $\gamma_{k}$. Therefore, $G$ acts freely on the set of all arcs $\left\{\gamma_{k}\right\}$. Moreover, the ends of any arc cannot lie in the same orbit of $G$. From this it follows that $\left|Z_{f_{1}}\right|=\left|Z_{f_{0}}\right|$ (mod $2|G|)$, i.e. $\operatorname{deg}_{G}\left(f_{0}\right)=\operatorname{deg}_{G}\left(f_{1}\right)$.

Theorem 1. Let $G$ be a finite group acting linearly on $\mathbb{R}^{n}$ with $\left(\mathbb{R}^{n}\right)^{G}=\{0\}$. Let $M^{n}$ be a closed connected PL free G-manifold. If there is a closed PL free $G$-manifold $N^{n}$ which is free $G$-cobordant to $M^{n}$ and a continuous equivariant transversal to zeros $h: N^{n} \rightarrow \mathbb{R}^{n}$ with $\operatorname{deg}_{G}(h)=1$, then for any continuous equivariant $f: M^{n} \rightarrow \mathbb{R}^{n}$ the zero set $Z_{f}$ is not empty.

Proof. Let $\operatorname{deg}_{G}(h)=1$. Suppose that $Z_{f}=\emptyset$. Since $M$ is compact, there is an $\varepsilon>0$ such that $\|f(x)\| \geq \varepsilon$ for all $x \in M$. From Lemma 3 it follows that there exists a generic $\tilde{f}$ such that $\|f(x)-\tilde{f}(x)\| \leq \varepsilon / 2$ for all $x \in M$. Then $\|\tilde{f}(x)\| \geq \varepsilon / 2$ 
for all $x \in M$ and $Z_{\tilde{f}}=\emptyset$. Therefore, $\operatorname{deg}_{G}(\tilde{f})=0$. On the other hand, Lemma 5 implies that $0=\operatorname{deg}_{G}(\tilde{f})=\operatorname{deg}_{G}(h)=1$, a contradiction.

Remark. Actually, Lemma 5 immediately implies that the assumption in the theorem: There is a free $G$-manifold $N^{n}$ which is free $G$-cobordant to $M^{n}$ and a continuous equivariant transversal to zeros $h: N^{n} \rightarrow \mathbb{R}^{n}$ with $\operatorname{deg}_{G}(h)=1$ is equivalent to the following statement: There is a continuous equivariant transversal to zeros map $h: M^{n} \rightarrow \mathbb{R}^{n}$ with $\operatorname{deg}_{G}(h)=1$. However, since the assumption in the theorem is more general, it sometimes can be more easily checked for $N$ which are free $G$-cobordant to $M$. (For instance, see our proof of Theorem 2.)

\section{BUT MANIFOLDS}

In this section, we consider the classical case $G=\mathbb{Z}_{2}$. Let $M$ be a closed PLmanifold with a free simplicial involution $T: M \rightarrow M$, i.e. $T^{2}(x)=x$ and $T(x) \neq x$ for all $x \in M$. For any $\mathbb{Z}_{2}$-manifold $(M, T)$ we say that a map $f: M^{m} \rightarrow \mathbb{R}^{n}$ is antipodal (or equivariant) if $f(T(x))=-f(x)$.

Definition. We say that a closed PL free $\mathbb{Z}_{2}$-manifold $\left(M^{n}, T\right)$ is a BUT (BorsukUlam Type) manifold if for any continuous $g: M^{n} \rightarrow \mathbb{R}^{n}$ there is a point $x \in M$ such that $g(T(x))=g(x)$. Equivalently, if a continuous map $f: M^{n} \rightarrow \mathbb{R}^{n}$ is antipodal, then $Z_{f}$ is not empty.

Let us recall several facts about $\mathbb{Z}_{2}$-cobordisms which are related to our main theorem in this section. We write $\mathfrak{N}_{n}$ for the group of unoriented cobordism classes of $n$-dimensional manifolds. Thom's cobordism theorem says that the graded ring of cobordism classes $\mathfrak{N}_{*}$ is $\mathbb{Z}_{2}\left[x_{2}, x_{4}, x_{5}, x_{6}, x_{8}, x_{9}, \ldots\right]$ with one generator $x_{k}$ in each degree $k$ not of the form $2^{i}-1$. Note that $x_{2 k}=\left[\mathbb{R} \mathbb{P}^{2 k}\right]$.

Let $\mathfrak{N}_{*}\left(\mathbb{Z}_{2}\right)$ denote the unoriented cobordism group of free involutions. Then $\mathfrak{N}_{*}\left(\mathbb{Z}_{2}\right)$ is a free $\mathfrak{N}_{*}$-module with basis $\left[\mathbb{S}^{n}, A\right], n \geq 0$, where $\left[\mathbb{S}^{n}, A\right]$ is the cobordism class of the antipodal involution on the $n$-sphere [5, Theorem 23.2]. Thus, each $\mathbb{Z}_{2^{-}}$ manifold can be uniquely represented in $\mathfrak{N}_{n}\left(\mathbb{Z}_{2}\right)$ in the form

$$
[M, T]=\sum_{k=0}^{n}\left[V^{k}\right]\left[\mathbb{S}^{n-k}, A\right] .
$$

Theorem 2. Let $M^{n}$ be a closed connected PL-manifold with a free simplicial involution $T$. Then the following statements are equivalent:

(a) $M$ is a BUT manifold.

(b) $M$ admits an antipodal continuous transversal to zeros map $h: M^{n} \rightarrow \mathbb{R}^{n}$ with $\operatorname{deg}_{\mathbb{Z}_{2}}(h)=1$.

(c) $\left[M^{n}, T\right]=\left[\mathbb{S}^{n}, A\right]+\left[V^{1}\right]\left[\mathbb{S}^{n-1}, A\right]+\ldots+\left[V^{n}\right]\left[\mathbb{S}^{0}, A\right]$ in $\mathfrak{N}_{n}\left(\mathbb{Z}_{2}\right)$.

Proof. (1) First we prove that (c) is equivalent to (b). Let

$$
[M, T]=\left[V^{0}\right]\left[\mathbb{S}^{n}, A\right]+\left[V^{1}\right]\left[\mathbb{S}^{n-1}, A\right]+\ldots+\left[V^{n}\right]\left[\mathbb{S}^{0}, A\right] .
$$

Denote by

$$
N:=V^{1} \times \mathbb{S}^{n-1} \sqcup \ldots \sqcup V^{n} \times \mathbb{S}^{0} .
$$

Note that for any $k$, where $0 \leq k<n$, the standard antipodal embedding of $\mathbb{S}^{k}$ into $\mathbb{R}^{n}$ has no zeros. This implies that there is a generic antipodal $p: N^{n} \rightarrow \mathbb{R}^{n}$ with $Z_{p}=\emptyset$. If $\left[V^{0}\right]=0$, then $M$ is free $\mathbb{Z}_{2}$-cobordant to $N$. Therefore, Lemma 5 
yields that for any generic antipodal $f: M \rightarrow \mathbb{R}^{n}$ we have $\operatorname{deg}_{\mathbb{Z}_{2}}(f)=\operatorname{deg}_{\mathbb{Z}_{2}}(p)=0$. This contradicts (b), and thus $\left[V^{0}\right]=1 \in \mathbb{Z}_{2}$.

On the other hand, if $\left[V^{0}\right]=1$, then $M$ is free $\mathbb{Z}_{2}$-cobordant to $L:=\mathbb{S}^{n} \sqcup N$. Take any generic antipodal $h: M^{n} \rightarrow \mathbb{R}^{n}$. Since for any generic antipodal $g: L \rightarrow \mathbb{R}^{n}$ we have $\operatorname{deg}_{\mathbb{Z}_{2}}(g)=1$, it again follows from Lemma 5 that $\operatorname{deg}_{\mathbb{Z}_{2}}(h)=1$.

(2) Theorem 1 yields: (b) implies (a).

(3) (a) implies (c). In fact, this follows from [12, Theorem 3] 2 This theorem yields that if $w_{1}^{n}\left(M / \mathbb{Z}_{2}\right)=0 \in H^{n}\left(M / \mathbb{Z}_{2}, \mathbb{Z}_{2}\right)$, then there is an antipodal continuous map $h: M^{n} \rightarrow \mathbb{S}^{n-1}$, i.e. $Z_{h}=\emptyset$. Therefore, $w_{1}^{n}\left(M / \mathbb{Z}_{2}\right) \neq 0$. This holds if and only if we have (c).

Remark. Actually, the list of equivalent versions in Theorem 2 can be continued. Tucker's lemma is a discrete version of the Borsuk-Ulam theorem:

Let $\Lambda$ be any equivariant triangulation of $\mathbb{S}^{n}$. Let

$$
L: V(\Lambda) \rightarrow\{+1,-1,+2,-2, \ldots,+n,-n\}
$$

be an equivariant (or Tucker) labelling, i.e. $L(T(v))=-L(v)$. Then there exists a complementary edge in $\Lambda$ such that its two vertices are labelled by opposite numbers (see [9, Theorem 2.3.1]).

Let $M$ be as in Theorem 2] Using the same arguments as in [9, Theorem 2.3.2], it can be proved that the following statement is equivalent to (a):

(d) For any equivariant labelling of an equivariant triangulation of $M$ there is a complementary edge.

In fact, any equivariant labelling $L$ defines a simplicial map $f_{L}: M \rightarrow \mathbb{R}^{n}$. Indeed, let $e_{1}, \ldots, e_{n}$ be an orthonormal basis of $\mathbb{R}^{n}$. We define $f_{L}: \Lambda \rightarrow \mathbb{R}^{n}$ for $v \in V(\Lambda)$ by $f_{L}(v)=e_{i}$ if $L(v)=i$ and $f_{L}(v)=-e_{i}$ if $L(v)=-i$. In the paper 11, it is shown that $M$ is a BUT manifold if and only if

(e) There exist an equivariant triangulation $\Lambda$ of $M$ and an equivariant labelling of $V(\Lambda)$ such that $f_{L}: \Lambda \rightarrow \mathbb{R}^{n}$ is transversal to zeros and the number of complementary edges is $4 k+2$, where $k=0,1,2, \ldots$.

Lyusternik and Shnirel'man proved in 1930 that for any cover $F_{1}, \ldots, F_{n+1}$ of the sphere $\mathbb{S}^{n}$ by $n+1$ closed sets, there is at least one set containing a pair of antipodal points (that is, $F_{i} \cap\left(-F_{i}\right) \neq \emptyset$ ). Equivalently, for any cover $U_{1}, \ldots, U_{n+1}$ of $\mathbb{S}^{n}$ by $n+1$ open sets, there is at least one set containing a pair of antipodal points [9, Theorem 2.1.1]. By the same arguments it can be shown that $M$ is a BUT manifold if and only if

(f) $M$ is a Lyusternik-Shnirel'man type manifold; i.e. for any cover $F_{1}, \ldots, F_{n+1}$ of $M^{n}$ by $n+1$ closed (respectively, by $n+1$ open) sets, there is at least one set containing a pair $(x, T(x))$.

Denote by $\operatorname{cat}(X)$ the Lyusternik-Shnirel'man category of a space $X$, i.e. the smallest $m$ such that there exists an open covering $U_{1}, \ldots, U_{m+1}$ of $X$ with each $U_{i}$ contractible to a point in $X$. It is not hard to prove the last statement in this remark:

(g) $M$ is a BUT manifold if and only if $\operatorname{cat}(M / T)=\operatorname{cat}\left(\mathbb{R} \mathbb{P}^{n}\right)=n$.

Usually, it is not easy to find $\operatorname{cat}(X)$. Here, Theorems 2-4 yield interesting possibilities to find lower bounds for $\operatorname{cat}(M / G)$.

\footnotetext{
${ }^{2}$ I would like to thank Alexey Volovikov, who noted this theorem. He as well as Pavle Blagojević and Roman Karasev also sent me another proof of [12, Theorem 3].
} 
Corollary 1. Let $M$ be any closed manifold. Then for the connected sum $M \# M$ there exists a "centrally symmetric" free involution such that $M \# M$ is a BUT manifold.

Proof. The Whitney embedding theorem states that any smooth or simplicial $n$ dimensional manifold can be embedded in Euclidean $2 n$-space. Consider an embedding of $M$ in $\mathbb{R}^{2 n}$ with coordinates $\left(x_{1}, \ldots, x_{2 n}\right)$. Let $M_{-}$(respectively, $M_{+}$) denote the set of points in $M \subset \mathbb{R}^{2 n}$ with $x_{1}<0$ (respectively, $x_{1}>0$ ). Let $M_{0}$ be the set of points in $M$ with $x_{1}=0$. Without loss of generality we can assume that $M$ is embedded in $\mathbb{R}^{2 n}$ in such a way that $M_{-}$is homeomorphic to an open $n$-ball and $M_{0}$ is a sphere $x_{1}^{2}+\ldots+x_{n+1}^{2}=1$ with $x_{n+2}=\ldots=x_{2 n}=0$. Then the central symmetry $s(s(x)=-x)$ is well defined on $X:=s\left(M_{+}\right) \cup M_{0} \cup M_{+}$as a free involution, and $X$ is homeomorphic to $M \# M$. Consider the projection $h$ of $X$ onto the $n$-plane $x_{n+1}=\ldots=x_{2 n}=0$, i.e. $h\left(x_{1}, \ldots, x_{2 n}\right)=\left(x_{1}, \ldots, x_{n}\right)$. Since $Z_{h} \subset M_{0}$, we have $Z_{h}=Z_{t}$, where $t:=\left.h\right|_{M_{0}}$. On the other hand, $t: M_{0} \rightarrow \mathbb{R}^{n}$ is an orthogonal projection of $\mathbb{S}^{n}$. Thus, $\left|Z_{h}\right|=2$ and Theorem 2 (b) implies that $M \# M$ is a BUT manifold.

\section{OBSTRUCtions FOR $G$-MAPS IN COBORDisms}

In the previous sections, we considered equivariant maps $f: M^{m} \rightarrow \mathbb{R}^{n}$ with $m=n$. Now we extend this approach to the case $m \geq n$.

Consider closed PL manifolds with an $H$-structure (such as an orientation). One can define a "cobordism with $H$-structure", but there are various technicalities. In each particular case, cobordism is an equivalence relation on manifolds. A basic question is to determine the equivalence classes for this relationship, called the cobordism classes of manifolds. These form a graded ring called the cobordism ring $\Omega_{*}^{H}$, with grading by dimension, addition by disjoint union, and multiplication by Cartesian product.

Let $\Omega_{*}^{H}(G)$ denote the PL cobordism group with $H$-structure of free simplicial actions of a finite group $G$. Let $\rho: G \rightarrow \mathrm{GL}(n, \mathbb{R})$ be a representation of a group $G$ on $\mathbb{R}^{n}$ which also has $H$-structure. Lemma 4 shows that for a generic simplicial equivariant map $f: M^{m} \rightarrow \mathbb{R}^{n}$ the cobordism class of the manifold $Z_{f}$ is uniquely defined up to cobordisms and so well defines a homomorphism

$$
\mu_{\rho}^{G}: \Omega_{m}^{H}(G) \rightarrow \Omega_{m-n}^{H}(G) .
$$

Remark. Note that the homomorphism $\mu_{\rho}^{G}: \Omega_{m}^{H}(G) \rightarrow \Omega_{m-n}^{H}(G)$ depends only on a representation of a group $G$ on $\mathbb{R}^{n}$. For some groups, this homomorphism is known in algebraic topology. For instance, if $G=\mathbb{Z}_{2}$ and $H=O$ (unoriented cobordisms, i.e. $\Omega_{*}^{O}\left(\mathbb{Z}_{2}\right)=\mathfrak{N}_{*}\left(\mathbb{Z}_{2}\right)$ ), then $\mu=\Delta^{k}$, where $\Delta$ is called the Smith homomorphism. Conner and Floyd [5, Theorem 26.1] give the following definition of $\Delta$ : Let $T$ be a free involution on a closed manifold $M$. For $n \geq m$ there exists an antipodal generic map $f: M^{m} \rightarrow \mathbb{S}^{n}$. Let $X^{m-1}=f^{-1}\left(\mathbb{S}^{n-1}\right)$. Then the map $\Delta: \Omega_{m}^{O}\left(\mathbb{Z}_{2}\right) \rightarrow \Omega_{m-1}^{O}\left(\mathbb{Z}_{2}\right)$ which is defined by $\left[M^{m}, T\right] \rightarrow\left[X^{m-1},\left.T\right|_{X}\right]$ is a homomorphism and $\Delta$ does not depend on $n$ and $f$.

The invariant $\mu_{\rho}^{G}$ is an obstruction for the existence of equivariant maps $f$ : $M \rightarrow \mathbb{R}^{n} \backslash\{0\}$. Namely, we have the following theorem.

Theorem 3. Let $M^{m}$ be a closed $P L G$-manifold with a free action $\tau$. Let $\rho$ be a linear action of $G$ on $\mathbb{R}^{n}$. Let us assume that actions, manifolds, and maps are with 
$H$-structure. Suppose that $\mu_{\rho}^{G}([M, \tau]) \neq 0$ in $\Omega_{m-n}^{H}(G)$. Then for any continuous equivariant map $f: M^{m} \rightarrow \mathbb{R}^{n}$ the set of zeros $Z_{f}$ is not empty.

Proof. Actually the proof of the theorem and of the corollary are almost word for word the same as the proof of Theorem 1. Let us suppose that $f^{-1}(0)=\emptyset$. Since $M$ is compact, there is an $\varepsilon>0$ such that $\|f(x)\| \geq \varepsilon$ for all $x \in M$. From Lemma 1 and Lemma 3, it follows that there exists a generic $h$ such that $\|f(x)-h(x)\| \leq \varepsilon / 2$ for all $x \in M$. By Lemma 4 we have $\mu_{\rho}^{G}([M, \tau])=\left[Z_{h}\right]_{G} \neq 0$ in $\Omega_{m-n}^{H}(G)$. Then $Z_{h}$ is a submanifold of $M$ of dimension $m-n$.

Since $Z_{h} \neq \emptyset$, there is an $x \in M$ such that $h(x)=0$. Thus, $\varepsilon / 2 \geq\|f(x)-h(x)\|=$ $\|f(x)\| \geq \varepsilon>0$, a contradiction.

Corollary 2. Let manifolds, actions, and maps be as above. Suppose that there is a continuous equivariant transversal (in zeros) map $h: M^{m} \rightarrow \mathbb{R}^{n}$ such that the set $Z_{h}$ is a closed submanifold in $M$ of codimension $n$ and $\left[Z_{h}\right]_{G} \neq 0$ in $\Omega_{m-n}^{H}(G)$. Then for any continuous equivariant map $f: M \rightarrow \mathbb{R}^{n}$ the set of zeros $Z_{f}$ is not empty.

Proof. By Lemma 3, for any $\varepsilon>0$ there is a generic map $a_{\varepsilon}$ such that $\| a_{\varepsilon}(x)-$ $h(x) \| \leq \varepsilon$ for all $x \in M$. If $\varepsilon \rightarrow 0$, then $Z_{\varepsilon}:=a_{\varepsilon}^{-1}(0) \rightarrow Z$. This implies that for a sufficiently small $\varepsilon$, there is a homeomorphism between $Z_{\varepsilon}$ and $Z_{h}$. Therefore, $\mu_{\rho}^{G}([M, \tau])=\left[Z_{h}\right]_{G} \neq 0$ in $\Omega_{m-n}^{H}(G)$.

Let $G=\mathbb{Z}_{2}$ and $\rho(x)=-x$. Denote by $\mu_{n}:=\mu_{\rho}^{\mathbb{Z}_{2}}$. Then we have

$$
\mu_{n}: \mathfrak{N}_{m}\left(\mathbb{Z}_{2}\right) \rightarrow \mathfrak{N}_{m-n}\left(\mathbb{Z}_{2}\right)
$$

If $u \in \mathfrak{N}_{m}\left(\mathbb{Z}_{2}\right)$ is given explicitly, then we can easily find $\mu_{n}(u)$.

\section{Lemma 6.}

$$
\mu_{n}\left(\left[M^{m}, T\right]\right)=\mu_{n}\left(\sum_{k=0}^{m}\left[V^{k}\right]\left[\mathbb{S}^{m-k}, A\right]\right)=\sum_{k=0}^{m-n}\left[V^{k}\right]\left[\mathbb{S}^{m-n-k}, A\right] .
$$

Proof. Since $Z_{f_{i}}=F^{-1}(0) \cap M_{i}$ in Lemma 4 are cobordant to each other, we can consider the standard projection $\operatorname{Pr}: \mathbb{S}^{d} \rightarrow \mathbb{R}^{n}$. Then $\operatorname{Pr}^{-1}(0)=\mathbb{S}^{d-n}$. This yields $\mu_{n}\left(\left[\mathbb{S}^{d}, A\right]\right)=\left[\mathbb{S}^{d-n}, A\right]$. It is clear that the lemma follows from this equality.

Lemma 6 and Theorem 3 imply the following corollary.

Corollary 3. Let $\left[M^{m}, T\right]=\left[V^{m-n}\right]\left[\mathbb{S}^{n}, A\right]+\left[V^{m-n+1}\right]\left[\mathbb{S}^{n-1}, A\right]+\ldots+\left[V^{m}\right]\left[\mathbb{S}^{0}, A\right]$ in $\mathfrak{N}_{m}\left(\mathbb{Z}_{2}\right)$ with $\left[V^{m-n}\right] \neq 0$ in $\mathfrak{N}_{m-n}$. Then for any antipodal continuous map $f: M^{m} \rightarrow \mathbb{R}^{n}$ the set $Z_{f}$ is not empty.

This corollary can be extended for the case $G=\left(\mathbb{Z}_{2}\right)^{k}=\mathbb{Z}_{2} \times \ldots \times \mathbb{Z}_{2}$. Note that $\mathfrak{N}_{*}\left(\left(\mathbb{Z}_{2}\right)^{k}\right)=\mathfrak{N}_{*}\left(\mathbb{Z}_{2}\right) \otimes \ldots \otimes \mathfrak{N}_{*}\left(\mathbb{Z}_{2}\right)$ [5. Sec. 29]. In other words, $\mathfrak{N}_{*}\left(\left(\mathbb{Z}_{2}\right)^{k}\right)$ is a free $\mathfrak{N}_{*}$-module with generators $\left\{\gamma_{i_{1}} \otimes \ldots \otimes \gamma_{i_{k}}\right\}, i_{1}, \ldots, i_{k}=0,1, \ldots$, where $\gamma_{i}:=\left[\mathbb{S}^{i}, A\right] \in \mathfrak{N}_{i}\left(\mathbb{Z}_{2}\right)$.

Corollary 4. Let $M$ be a closed PL-manifold with a free action $\Psi$ of $\left(\mathbb{Z}_{2}\right)^{k}$. Let $\left[M^{m}, \Psi\right]=\sum\left[V^{i_{1}, \ldots, i_{k}}\right] \gamma_{i_{1}} \otimes \ldots \otimes \gamma_{i_{k}}$ in $\mathfrak{N}_{m}\left(\left(\mathbb{Z}_{2}\right)^{k}\right)$ with $\left[V^{i_{1}, \ldots, i_{k}}\right] \neq 0$ in $\mathfrak{N}_{*}$. Consider $\left(\mathbb{Z}_{2}\right)^{k}$ with generators $T_{1}, \ldots, T_{k}$ acting on $\mathbb{R}^{i_{1}} \oplus \ldots \oplus \mathbb{R}^{i_{k}}$ by $T_{\ell}(x)=-x$ for $x \in \mathbb{R}^{i_{\ell}}$. Then for any continuous equivariant map $f: M^{m} \rightarrow \mathbb{R}^{i_{1}} \oplus \ldots \oplus \mathbb{R}^{i_{k}}$ the set $Z_{f}$ is not empty. 
Let $\tilde{G}(4,2)$ be the oriented Grassmann manifold, that is, the space of all oriented 2-dimensional subspaces of $\mathbb{R}^{4}$. Note that $\mathbb{Z}_{2} \times \mathbb{Z}_{2}$ acts on $\tilde{G}(4,2)$, where for $x \in$ $\tilde{G}(4,2), T_{1}(x)$ changes the orientation of $x$ and $T_{2}(x)$ is the oriented orthogonal complement of an oriented 2-plane $x$ in $\mathbb{R}^{4}$.

It is well known that $\tilde{G}(4,2)$ is diffeomorphic to $\mathbb{S}^{2} \times \mathbb{S}^{2}$ [7, 3.2.3]. For $(u, v) \in$ $\mathbb{S}^{2} \times \mathbb{S}^{2}$, we have $T_{1}(u, v)=(-u,-v)$ and $T_{2}(u, v)=(u,-v)$. Then for $\Psi=\left(T_{1}, T_{2}\right)$ we have $[\tilde{G}(4,2), \Psi]=\gamma_{2} \otimes \gamma_{2}$ in $\mathfrak{N}_{4}\left(\mathbb{Z}_{2} \times \mathbb{Z}_{2}\right)$. Then Corollary 4 (or Theorem 1) yields:

Corollary 5. Let $\mathbb{Z}_{2} \times \mathbb{Z}_{2}$ act on $\mathbb{R}^{4}=\mathbb{R}^{2} \oplus \mathbb{R}^{2}$ by $T_{1}(u, v)=(-u,-v)$ and $T_{2}(u, v)=(u,-v)$. Then for any equivariant continuous $f: \tilde{G}(4,2) \rightarrow \mathbb{R}^{4}$ the set $Z_{f}$ is not empty.

\section{ACKNOWLEDGMENTS}

I wish to thank Arseniy Akopyan, Imre Bárány, Pavle Blagojević, Mike Field, Jiří Matoušek, Alexey Volovikov, and Günter Ziegler for helpful discussions and comments. I am most grateful to Roman Karasev and to the anonymous referee for several critical comments and corrections.

\section{REFERENCES}

[1] I. Bárány, Borsuk's theorem through complementary pivoting, Math. Programming, 18 (1980), 84-88. MR557116 (81d:90099)

[2] E. Bierstone, General position of equivariant maps, Trans. Amer. Math. Soc., 234 (1977), 447-466. MR0464287 (57:4221)

[3] G. E. Bredon, Introduction to compact transformation groups, Academic Press, 1972. MR0413144 (54:1265)

[4] P. E. Conner and E. E. Floyd, Fixed point free involutions and equivariant maps, Bull. Amer. Math. Soc., 66 (1960), 416-441. MR0163310(29:613)

[5] P. E. Conner and E. E. Floyd, Differentiable periodic maps, Springer-Verlag, 1964. MR0176478(31:750)

[6] M. J. Field, Transversality in G-manifolds, Trans. Amer. Math. Soc., 231 (1977), 429-450. MR0451276 (56:9563)

[7] D. B. Fuks and V. A. Rokhlin, Beginner's course in topology. Geometric chapters, SpringerVerlag, Berlin-New York, 1984. MR759162 (86a:57001)

[8] S. Illman, Smooth equivariant triangulations of $G$-manifolds for $G$ a finite group, Math. Ann., 233 (1978), 199-220. MR0500993 (58:18474)

[9] J. Matoušek, Using the Borsuk-Ulam theorem, Springer-Verlag, Berlin, 2003. MR 1988723 (2004i:55001)

[10] M. D. Meyerson and A. H. Wright, A new and constructive proof of the Borsuk-Ulam theorem, Proc. Amer. Math. Soc., 73 (1979), 134-136. MR512075(80e:55006)

[11] O. R. Musin, Sperner and Tucker's lemmas and its generalizations, in preparation.

[12] D. Repovš and A. Skopenkov, On projected embeddings and desuspending the $\alpha$-invariant, Topology and its Applications, 124 (2002) 69-75. MR1926135 (2003g:57046)

[13] H. Steinlein, Borsuk's antipodal theorem and its generalizations and applications: A survey, Méth. Topol. en Anal. Non Linéaire, Sém. Math. Sup., 95 (1985), Presses Univ. Montréal, Montreal, Quebec, 166-235. MR801938 (86k:55002)

[14] A. S. Svarc, The genus of a fibered space, Trudy Moskov. Mat. Obsc., 10 (1961), 217-272; and 11 (1962), 99-126 (Russian). English translation in Amer. Math. Soc. Translat., II. Ser., 55 (1966), 49-140. MR0154284 (27:4233)

Department of Mathematics, University of Texas at Brownsville, 80 Fort Brown, Brownsville, Texas 78520

E-mail address: oleg.musin@utb.edu 